\title{
Post-Malaria Anemia Is Rare in Malawian Children with Cerebral Malaria
}

\author{
Geoffrey Guenther, ${ }^{1}$ Alexuse M. Saidi, ${ }^{2}$ Rima Izem, ${ }^{3,4}$ Karl Seydel, ${ }^{2,5}$ and Douglas G. Postels ${ }^{2,6 *}$ \\ ${ }^{1}$ Department of Pediatrics, Children's National Medical Center, Washington, District of Columbia; ${ }^{2}$ Blantyre Malaria Project, University of Malawi \\ College of Medicine, Blantyre, Malawi; ${ }^{3}$ Division of Biostatistics and Study Methodology, Children's National Research Institute, Washington, \\ District of Columbia; ${ }^{4}$ Division of Epidemiology, The George Washington University School of Public Health, Washington, District of Columbia; \\ ${ }^{5}$ Department of Osteopathic Medical Specialties, College of Osteopathic Medicine, Michigan State University, East Lansing, Michigan; ${ }^{6}$ Division of \\ Neurology, The George Washington University/Children's National Medical Center, Washington, District of Columbia
}

\begin{abstract}
Artesunate therapy for severe malaria syndromes has been associated with post-treatment hemolysis and anemia. We defined post-malaria anemia as any decrease in hematocrit between the index hospitalization for severe malaria and 1 month after. We determined the incidence and severity of post-malaria anemia in Malawian children surviving cerebral malaria $(\mathrm{CM})$ by analyzing hospital and follow-up data from a long-standing study of CM pathogenesis. Children enrolled before 2014 and treated with quinine $(N=258)$ were compared with those admitted in 2014 and after, and treated with artesunate $(N=235)$. The last hematocrit value obtained during hospitalization was compared with the 1 -month post-hospitalization hematocrit value. The overall rate of a post-hospitalization decrease in hematocrit in children surviving CM was $5.3 \%$ (11 of 235 or $4.7 \%$ for quinine, 15 of 258 or $5.8 \%$ for artesunate; odds ratio, 3.23 [0.88, 18.38]); no patients with a decrease in hematocrit were symptomatic, and none required transfusion after hospitalization. Of the 26 children who had a decrease in hematocrit 1 month after hospitalization, $23.1 \%$ had evidence of a new malaria infection. When children treated with quinine and artesunate were combined, a higher hematocrit level on admission, lower quantitative histidine-rich protein level, and splenomegaly were associated independently with post-malaria anemia. In African survivors of CM, post-malaria anemia is rare, mild, and unassociated with the anti-malarial treatment received.
\end{abstract}

\section{INTRODUCTION}

Malaria continues to be the most prevalent and clinically important human parasitic disease. One third of the world's population is at risk, with 228 million cases and more than 400,000 deaths each year. Children younger than 5 years have the highest burden of malaria-related mortality, accounting for two thirds of all deaths. ${ }^{1}$

Severe malaria is defined as end-organ dysfunction associated with malaria infection, and it requires urgent treatment and hospitalization. After clinical trials of quinine versus artesunate in the treatment of severe malaria showed a significant reduction in mortality in those treated with artesunate (with no increase in neurological deficits in survivors), the WHO changed its recommendation for the anti-malarial of choice in the treatment of severe malaria from quinine to artesunate. ${ }^{2,3}$ The adoption of artesunate was rapid, and is now the recommended first-line anti-malarial for severe malaria syndromes in almost all countries in Africa. ${ }^{1}$

Although artesunate decreases mortality, its rollout revealed adverse events not seen with previously used anti-malarials. These included delayed hemolysis, observed days to weeks after completion of therapy. The mechanism of hemolysis has recently been elucidated: artesunate-killed parasites are expelled from red blood cells, which reseal rather than lyse, leading to pitting. Pitted erythrocytes remain in the circulation but have a significantly diminished life span, eventually being cleared by the spleen.

Early retrospective studies in travelers returning from malaria endemic areas showed that post-artesunate hemolysis $(\mathrm{PAH})$, defined as $\mathrm{a} \geq 10 \%$ decrease in hemoglobin concentration after completion of artesunate therapy, occurs in $13 \%{ }^{4}$ to $15 \%{ }^{5}$ of patients. A prospective study of returning travelers revealed an even higher proportion, with PAH in $22 \%$ of patients studied. ${ }^{6}$ Hemolysis may be severe, and $73 \%$ of patients required blood transfusions. ${ }^{4}$ Clearly, if PAH were

*Address correspondence to Douglas G. Postels, Division of Neurology, Children's National Medical Center, 111 Michigan Ave. NW, Washington, DC 20010. E-mail: dpostels@childrensnational.org prevalent and severe in endemic areas, this could have significant public health implications, because blood transfusion may not be as readily available as in high-income countries. In addition, because PAH can occur up to 3 weeks after treatment, in areas where transportation may not be readily available, patients returning to their homes after hospitalization may be at personal risk of a delayed adverse outcome.

Recent studies of PAH incidence and severity have shifted their focus from returning travelers to African children (Supplemental Table S1). A prospective dual-center study on children with all forms of severe malaria in Ghana and Gabon showed $7 \%$ of children treated with artesunate experienced $\mathrm{PAH}$ on post-treatment day $14 .{ }^{7}$ After these results were published, the investigators enrolled additional patients, lowering the combined incidence rate to $5 \% .^{8} \mathrm{~A}$ prospective study of Ugandan children with multiple severe malaria syndromes showed no evidence of PAH, although laboratory tests were only performed on patients with clinical signs of anemia. ${ }^{9}$

Cerebral malaria $(\mathrm{CM})$ is the most lethal form of severe malaria. Its pathophysiology includes sequestration of parasitized red blood cells in the cerebral vasculature and evolving cerebral edema, accompanied by coma and other neurological abnormalities. In the only previously published study of post-treatment hemolysis that explicitly included patients with $\mathrm{CM}$, children in Mozambique with varying severe malaria syndromes were enrolled from 2003 to $2017 .{ }^{10}$ Of the 1,487 patients admitted who had a hematocrit result available at 1 month after admission, 1,333 were treated with quinine and 154 with artesunate. Of the cohort, $1.08 \%(16$ of 1,487$)$ had CM. The incidence of post-treatment hemolysis was the same comparing those treated with quinine and artesunate. Splenomegaly during admission was an independent risk factor for the development of post-treatment hemolysis. Significantly more patients required post-discharge blood transfusion after treatment with artesunate than quinine.

Other studies limiting enrollment and analysis to patients with a single severe malaria syndrome are rare. A randomized controlled clinical trial of quinine versus artesunate in Congolese children with the hyperparasitemic form of severe malaria determined that 
$5 \%$ of patients treated with artesunate had a significant decrease in hemoglobin 7 days after treatment; only one patient of the 109 who received artesunate required transfusion. ${ }^{11}$

As different severe malaria syndromes likely have differing dominant pathophysiology producing their most important clinical symptoms, it is possible that post-malaria anemia may be more common in one patient subpopulation compared with the overall group. In response, we performed a retrospective cohort study using data collected previously by a longstanding study of $\mathrm{CM}$ pathogenesis the Blantyre Malaria Project. We aimed to determine the incidence, severity, and risk factors for post-malaria anemia in children with $\mathrm{CM}$. We compared the likelihood of post-malaria anemia in children treated with quinine (before 2014) to those treated with artesunate (in 2014 and after).

\section{MATERIALS AND METHODS}

Clinical care and specimen collection. We included participants 6 months to 14 years of age, admitted between January 2010 and June 2019, with a clinical diagnosis of CM: Blantyre coma score $\leq 2$, asexual forms of Plasmodium falciparum on peripheral blood smear, and no other known etiology for coma (e.g., hypoglycemia, post-ictal state, or cerebrospinal fluid pleocytosis). The parent project was a long-standing study of CM pathogenesis that enrolled subjects at Queen Elizabeth Central Hospital in Blantyre, Malawi. The parent study underwent ethical review and was approved by the Institutional Review Boards of Michigan State University and the University of Malawi College of Medicine. At the time of enrollment into the parent study, caregivers consented to the secondary analysis of deidentified data, which were used in our research.

After clinical stabilization, patients were started on intravenous maintenance fluids and anti-malarials (quinine up to and including 2013, artesunate thereafter), and the highest level of supportive care available. Supportive care included nasogastric feeds beginning typically at 24 hours after admission, anti-convulsants for clinical seizures or nonconvulsive status epilepticus, and blood transfusions $(10 \mathrm{~mL} / \mathrm{kg}$ packed red blood cells or $20 \mathrm{~mL} / \mathrm{kg}$ whole blood) for circulatory instability resulting from hypovolemia. Mechanical ventilation was not available. Before and including 2013, quinine was given with an initial loading dose of $20 \mathrm{mg} / \mathrm{kg}$ infused over 4 hours, followed by three doses of $10 \mathrm{mg} / \mathrm{kg}$ every 12 hours. In 2014 and afterward, three doses of artesunate were administered per WHO-recommended dosing, with one modification. If a child remained unconscious 24 hours after hospital admission, artesunate was discontinued and therapy was switched to an artemisinin combination therapy (usually lumefantrine-artemether) for the subsequent 3 days at doses recommended by the Malawi Ministry of Health. If the child's mental status did not allow medication to be taken orally, a nasogastric tube was used for initiation or completion of enteral therapy until oral medication administration was safe.

Finger prick samples for determination of hematocrit were collected on admission and every 6 hours afterward until two consecutive blood smears for malaria parasites were negative. Capillary tubes for hematocrit were spun on a micro-hematocrit centrifuge (Hawksley, Sussex, UK) for 3 minutes and were then read using a Micro Haematocrit Reader (Hawksley, Sussex, UK).

At admission, additional testing was performed, including determination of serum glucose, quantitative histidine-rich protein 2 (qHRP2), and other research related laboratory studies. Survivors were seen 1 month after admission for physical and neurological exams, a blood smear for malaria parasites, and hematocrit determination.

Admission parasitemia was determined by manual counting. Both thick and thin blood smears were prepared for all participants at all time points. If initial review by laboratory technicians showed a high parasite burden, the thin smear was examined and the number of parasites per 500 red blood cells was counted. If a lower parasite burden was seen, the thick smear was analyzed and the number of parasites per 200 white blood cells was determined using methods described previously by the WHO. ${ }^{12}$ The admission circulating parasite density was calculated by standard methods, using either red blood cell or white blood cell counts obtained at admission. ${ }^{12}$

qHRP2 levels were determined on blood samples obtained at admission and stored at $-80^{\circ} \mathrm{C}$ until analyzed. Standardized recombinant HRP2 stock was titrated to produce a standard curve. A total of $100 \mu \mathrm{L}$ of unknown plasma samples were diluted at 1:500 in 0.1\% bovine serum albumin/phosphatebuffered saline (PBS) and were then added in duplicate to an anti-HRP2 precoated 96-well plate. The plate was incubated at $37^{\circ} \mathrm{C}$ for 1 hour, then washed with $1 \%$ Tween/PBS. A total of $100 \mu \mathrm{L}$ conjugated antibody was then added to the plate, incubation was repeated at $37^{\circ} \mathrm{C}$, and the plate was again washed. A total of $100 \mu \mathrm{L}$ of substrate was added and incubated for 15 minutes at room temperature in the dark. Reactions were stopped by adding $50 \mu \mathrm{L}$ stop solution. Plates were analyzed immediately using an ELx800 plate reader (Biotek Instruments, Winooski, VT) at a wavelength of $450 \mathrm{~nm}$. The HRP2 concentration in plasma was calculated using a standard curve generated from the recombinant HRP2 protein stock. Samples with results that fell outside the standard curve linear ranges were repeated with either the dilution factor increased (if the result was greater) or reduced (if the result was less) and the re-diluted samples were re-analyzed.

Statistical analysis. We defined post-malaria anemia as a decline in hematocrit at the 1-month follow-up compared with the last hematocrit measured before hospital discharge. Candidate risk factors for post-malaria anemia included several characteristics measured at admission: age, sex, Blantyre coma score, splenomegaly, glucose, peripheral parasitemia, qHRP2, and hematocrit. Summaries of these data for our cohort included means and SDs for continuous variables or counts and frequencies for categorical variables. We compared characteristics between quinine-treated and artesunate-treated patients who did and did not have post-malaria anemia using $t$ tests for continuous characteristics, or $x^{2}$ tests for categorical variables. We logarithmically transformed peripheral parasite densities and qHRP2 levels to stabilize the variance when making comparisons. To determine whether a candidate risk factor identified on univariate analyses $(P<0.10)$ was associated independently with a post-hospitalization decrease in hematocrit, we used multivariate logistic regression and estimated 95\% Cls with bootstrap. We explored whether the impact of each risk factor varied by the anti-malarial given for treatment by including an interaction term of each risk factor with the anti-malarial used in the multivariable model. In all our results, we considered a $P$ value less than 0.05 to show a statistically significant difference between groups. All analyses were performed using the $\mathrm{R}$ software package, version 4.0.2 (R Foundation for Statistical Computing, Vienna, Austria). 


\section{RESULTS}

Between 2010 and 2019, 706 patients were enrolled in the parent study and received either quinine $(N=371)$ or artesunate $(N=335)$ (Figure 1). Our analysis cohort (493 subjects: 235 treated with quinine and 258 with artesunate) included those subjects with hematocrit measures during hospitalization and at 1-month post-admission. We excluded from our analysis those with missing values in one of these measures.

Those who received quinine were younger, had higher admission glucose levels, higher rates of splenomegaly, higher qHRP2 levels, and lower admission hematocrit values compared with those who received artesunate (Table 1). Although there were more missing one-month post-admission PCV values for patients treated with quinine than artesunate (51.6\% missing in quinine vs. $39.9 \%$ for artesunate, $P=0.006$ ), excluded subjects were similar in demographics and baseline laboratory values compared to those included in the analyzed cohort (Table 2). For our main outcome of post-malaria anemia, 26 patients $(5.27 \%$ ) (quinine- and artesunate-treated patients combined) had a decrease in hematocrit when comparing their 1-month post-hospitalization hematocrit value to their last value recorded during hospitalization (Table 3). This rate of post-malaria anemia did not vary by the anti-malarial used for treatment $(P=0.718)$. For those with a post-admission decrease in hematocrit, the number of those with evidence of malaria re-infection (parasites present on microscopy at 1 month post-hospitalization) was not different in those treated with artesunate (5 of 15) compared with those treated with quinine ( 1 of 11$)(P=0.147)$. On univariate analysis, a higher hematocrit at hospital admission, a lower qHRP2, and the presence of splenomegaly were each associated with post-malaria anemia. In a multivariable logistic regression model including all three of these characteristics, a higher hematocrit at admission and lower qHRP2 value were associated independently with post-malaria anemia (Table 4). The independent association of qHRP2 and the presence of

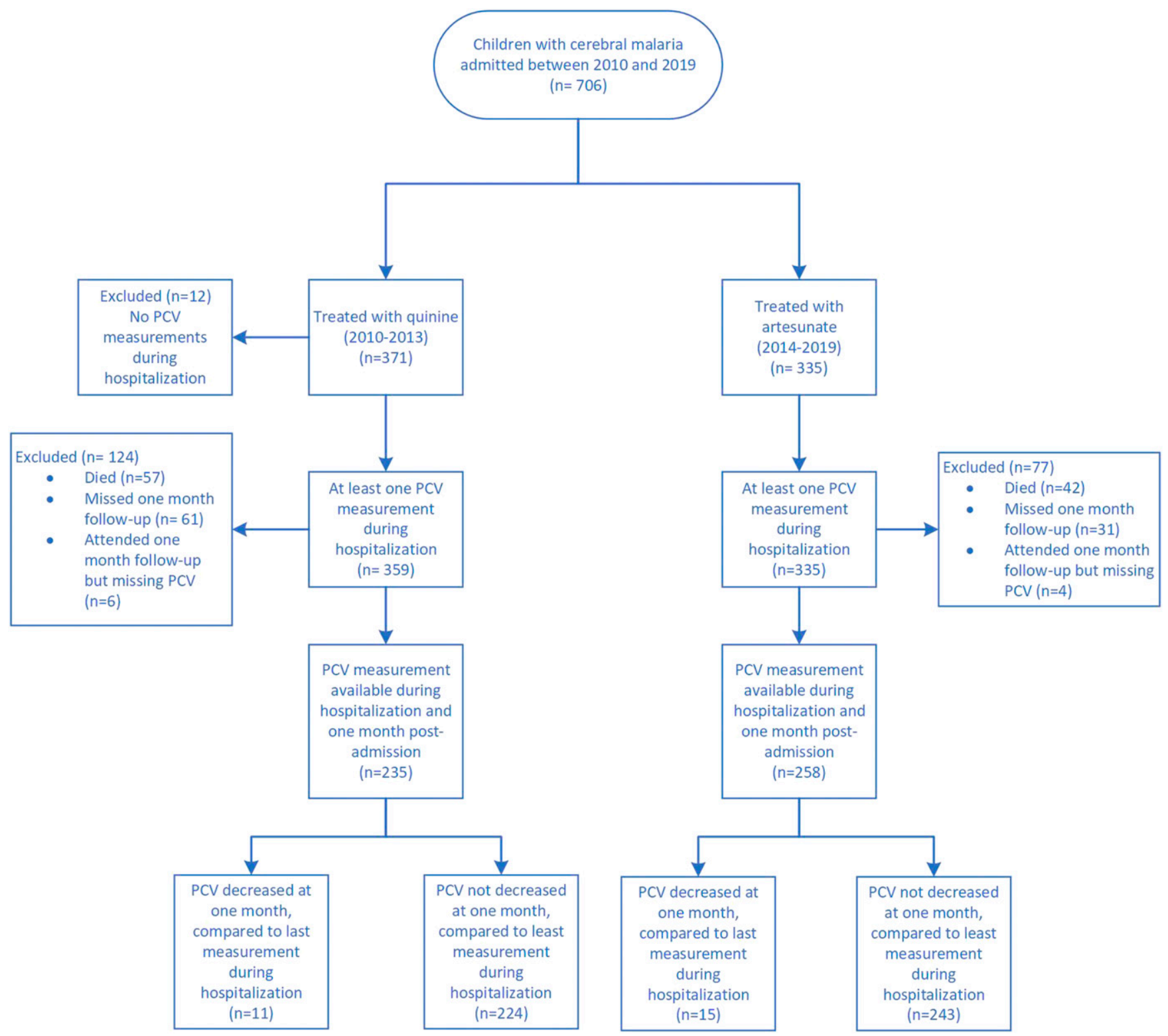

FIGURE 1. Study population. PCV = packed cell volume. This figure appears in color at www.ajtmh.org. 
TABLE 1

Demographic, clinical characteristics, and outcomes of children with cerebral malaria included in the study population (patients who survived to hospital discharge with hematocrit available at 1-month follow-up)

\begin{tabular}{|c|c|c|c|c|}
\hline Demographic, clinical, outcome measure & $\begin{array}{l}\text { Total analyzed cohort } \\
\qquad(N=493)\end{array}$ & $\begin{array}{l}\text { Quinine-treated, 2010-2013 } \\
\qquad(N=235)\end{array}$ & $\begin{array}{l}\text { Artesunate-treated, 2014-2019 } \\
\qquad(N=258)\end{array}$ & $P$ value \\
\hline Age (months), mean (SD) & $52.84(29.74)$ & $49.13(27.66)$ & $56.22(31.18)$ & 0.008 \\
\hline $\begin{array}{l}\text { Pre-hospital anti-malarial treatment, } N \\
\quad(\%)\end{array}$ & $467(94.7)$ & $225(95.7)$ & $242(93.8)$ & 0.461 \\
\hline Male, $N(\%)$ & $251(50.9)$ & $121(51.5)$ & $130(50.4)$ & 0.877 \\
\hline Splenomegaly, $N(\%)$ & $127(25.9)$ & 75 (31.9) & 52 (20.3) & 0.005 \\
\hline Blantyre coma score at admission, $N(\%)$ & & & & 0.323 \\
\hline 0 & $33(6.7)$ & $15(6.4)$ & $18(7.0)$ & \\
\hline 1 & $201(40.8)$ & $104(44.3)$ & 97 (37.6) & \\
\hline 2 & $259(52.5)$ & $116(49.4)$ & $143(55.4)$ & \\
\hline Glucose (mmoL/L), mean (SD) & $6.59(2.73)$ & $6.90(3.06)$ & $6.30(2.36)$ & 0.016 \\
\hline Hemoglobin (admission), mean (SD) & $7.64(2.47)$ & $7.28(2.35)$ & $7.88(2.04)$ & 0.003 \\
\hline Hematocrit (admission), mean (SD) & $23.28(6.79)$ & $22.63(7.00)$ & $23.88(6.56)$ & 0.041 \\
\hline $\begin{array}{l}\text { Hematocrit (last measured during } \\
\text { admission), mean (SD) }\end{array}$ & $23.61(5.23)$ & $23.13(5.16)$ & $24.05(5.28)$ & 0.050 \\
\hline Hematocrit (1 mo), mean (SD) & $35.00(4.66)$ & $35.13(4.14)$ & $34.88(5.10)$ & 0.556 \\
\hline Parasite count, mean (SD) & $148,844(362,765)$ & $188,132(467,344)$ & $115,282(235,735)$ & $0.001^{*}$ \\
\hline Parasite count, median (IQR) & $27,020(584-166,445)$ & $51,120(1,192-222,140)$ & $6,084(387-84,720)$ & $<0.0001 \dagger$ \\
\hline qHRP2, mean (SD) & $6,386(10,667)$ & $7,174(10,307)$ & $5,693(10,947)$ & $0.130^{\star}$ \\
\hline qHRP2, median (IQR) & $1,948(322-8,477)$ & $3,028(563-9,260)$ & $1,200(285-6,584)$ & $0.006 \dagger$ \\
\hline $\begin{array}{l}\text { Severe anemia at admission (hematocrit } \\
\quad<15 \%), N(\%)\end{array}$ & $48(9.7)$ & $27(11.5)$ & $21(8.1)$ & 0.359 \\
\hline Blood transfusion during admission, $N(\%)$ & $179(36.3)$ & $102(43.4)$ & $77(29.8)$ & 0.002 \\
\hline Outcome in survivors & & & & NS \\
\hline $\begin{array}{l}\text { Survived without neurological sequelae } \\
\text { at hospital discharge, } N(\%)\end{array}$ & $437(88.8)$ & $206(88.0)$ & $231(89.5)$ & \\
\hline $\begin{array}{l}\text { Survived with neurological sequelae at } \\
\text { hospital discharge, } N(\%)\end{array}$ & 55 (11.2) & $28(12.0)$ & 27 (10.5) & \\
\hline
\end{tabular}

post-malaria anemia depended on the anti-malarial used; this independent association was observed in quinine-treated patients but not in those treated with artesunate (Table 5, $P<$ 0.05 for the interaction term).

\section{DISCUSSION}

A post-hospitalization decrease in hematocrit is both infrequent and mild in children with CM. No patients in our cohort required blood transfusion between discharge and 1-month post-hospitalization, regardless of whether they were treated with quinine or artesunate. The likelihood of a post-hospitalization decrease in hematocrit was no greater with the use of artesunate than with quinine.

A significant hemolytic event after hospital discharge would be dangerous in malaria-endemic areas in Sub-Saharan Africa, which continue to struggle with maintaining an adequate and safe supply of blood products for transfusion. ${ }^{13}$ The combination of a hemolytic event superimposed on a background of chronic anemia may be even more dangerous. A recent study in rural Malawi showed a prevalence of non-malarial anemia in children of almost $60 \% .{ }^{14}$ Certainly, if there were a high incidence and severity of post-malaria hemolysis in African children treated with artesunate, this could offset some of the significant mortality

TABLE 2

Comparison of baseline demographic, clinical, and outcomes for patients excluded from analysis as a result of missing admission or post-discharge hematocrit values $(N=213)$ and those included in all analyses $(N=493)$

\begin{tabular}{lrr}
\hline Demographic or clinical characteristic & $\begin{array}{c}\text { Excluded as a result of missing hematocrit } \\
\text { measures }(N=213)\end{array}$ & $\begin{array}{c}\text { Included in all analyses } \\
(N=493)\end{array}$ \\
\hline Age (months), mean (SD) & $52.0(30.9)$ & $52.8(29.7)$ \\
Sex (male), $N(\%)$ & $115(54.0)$ & $251(50.9)$ \\
Blantyre coma score & & 0.729 \\
0 & $29(13.6)$ & $33(6.7)$ \\
1 & $90(42.3)$ & $201(40.8)$ \\
2 & $94(44.1)$ & $259(52.3)$ \\
qHRP2, mean (SD) & $6,973.3(10,117.5)$ & $6,386.0(10,666.8)$ \\
Parasite count, mean (SD) & $197,441.9(390,269.2)$ & $148,844.2(362,765.1)$ \\
Splenomegaly, $N(\%)$ & $62(29.1)$ & $127(25.9)$ \\
Outcome, $N$ (\%) & & 0.504 \\
$\quad$ Died & $101(48.6)$ & 0.100 \\
Survived with sequelae & $9(4.3)$ & 0.424 \\
Survived without sequelae & $98(47.1)$ & $55(11.2)$ \\
\hline
\end{tabular}


TABLE 3

Comparison of patients with no drop in PCV to those with a drop in PCV in the analysis cohort

\begin{tabular}{|c|c|c|c|}
\hline Demographic or clinical characteristic & No decline in PCV $(N=467)$ & Decline in PCV $(N=26)$ & $P$ value for difference \\
\hline Age (months), mean (SD) & $52.84(29.33)$ & $52.81(37.02)$ & 0.995 \\
\hline Male, $N(\%)$ & $234(50.1)$ & $17(65.4)$ & 0.188 \\
\hline $\begin{array}{l}\text { Duration of illness prior to hospital } \\
\text { admission (h), mean (SD) }\end{array}$ & $58.6(38.4)$ & 60.4 (22.3) & 0.66 \\
\hline Splenomegaly, $N(\%)$ & $125(26.9)$ & $2(7.7)$ & 0.052 \\
\hline Blantyre coma score at admission, $N(\%)$ & & & 0.277 \\
\hline 0 & $30(6.4)$ & $3(11.5)$ & \\
\hline 1 & $188(40.3)$ & $13(50.0)$ & \\
\hline 2 & $249(53.3)$ & $10(38.5)$ & \\
\hline Anti-malarial, $N(\%)$ & & & 0.718 \\
\hline Quinine & $224(48.0)$ & $11(43.3)$ & \\
\hline Artesunate & $243(52.0)$ & $15(57.7)$ & \\
\hline Hematocrit at admission, mean (SD) & $22.83(6.48)$ & $31.72(7.00)$ & $<0.001$ \\
\hline qHRP2, mean (SD) & $6,464(9,925)$ & $4,965(20,118)$ & $<0.001^{*}$ \\
\hline Parasite count, mean (SD) & $147,152(362,002)$ & $180,854(38,3884)$ & $0.806^{\star}$ \\
\hline Survived without sequelae, $N(\%)$ & $415(89.1)$ & $22(84.6)$ & 0.704 \\
\hline
\end{tabular}

benefit found in the clinical trials of artesunate versus quinine. We found that such events, when present in children with $\mathrm{CM}$, were rare and did not require therapeutic transfusion.

Although our results are consistent with other studies of $\mathrm{PAH}$ in African children with severe malaria syndromes (Supplemental Table S1), they are in stark contrast to studies of adult travelers returning from malaria endemic areas, which showed $\mathrm{PAH}$ in $13 \%$ to $22 \%$ of patients studied. ${ }^{4-6}$ The reasons for the difference in incidence rate between immunenaive travelers and African children was assessed in an earlier study. ${ }^{4}$ Children living in malaria-endemic areas, who have partial immunity to malaria as a result of repeated exposure, have lower levels of post-artesunate pitting and earlier erythropoiesis. Both these factors protect against the development of post-artesunate decreases in hematocrit.

In our study, an increase in qHRP2 was strongly associated with a decreased risk of the development of post-malaria anemia, particularly in patients treated with quinine. qHRP2 is a marker of total body parasite burden, reflecting both sequestered and circulating parasitized erythrocytes. In contrast, the concentration of circulating parasites was associated less strongly but had the same negative association; higher circulating parasites were associated with protection from the development of post-malaria anemia. The negative association was unexpected, as previous studies have found a greater likelihood of $\mathrm{PAH}$ in those with hyperparasitemia. ${ }^{7,8}$ Because detectable HRP2 may persist in the blood for 2 weeks after resolution of a malaria infection, it is possible that higher qHRP2 values in those with lower odds of post-malaria anemia reflect long-standing malaria infection, which may chronically

TABLE 4

Independent association of admission characteristics associated with post-admission hemolysis, results of multivariable logistic regression

\begin{tabular}{ll}
\hline \multicolumn{1}{c}{ Admission characteristic ${ }^{*}$} & Odds ratio $(95 \% \mathrm{Cl} \dagger)$ \\
\hline Hematocrit at admission & $9.44(3.00,33.01)$ \\
Ln(qHRP2) & $0.35(0.14,0.80)$ \\
Splenomegaly & $0.42(0.06,1.58)$ \\
\hline qHRP2 = quantitative histidine-rich protein 2. & \\
* qHRP2 was fit in the natural logarithm scale. Ln(qHRP2) and hematocrit at admission were \\
each rescaled (subtract overall mean and divide by two SDs) to be in the same scale. \\
$\quad$ † Ninety-five percent Cl with bootstrap.
\end{tabular}

stimulate erythropoiesis. Certainly, it is equally possible that the low event rate of post-admission decreases in hematocrit in our study population resulted in spurious, non-meaningful associations. Reasons for our findings are unclear and should be verified prospectively.

Many patients enrolled in our study received anti-malarial treatment prior to hospital presentation $(96 \%$ of those who received quinine versus $94 \%$ of those who received artesunate). Pre-treatment almost certainly lowered admission parasite densities compared with those found in medication-naive patients. qHRP2, a measure of total body parasite burden, is comparatively unaffected by pre-hospital anti-malarial administration. Further studies to evaluate the possibly non-linear relationship between circulating parasitemia and qHRP2 over the course of anti-malarial treatment are warranted.

Our study has several benefits compared with previously published studies of post-malaria anemia in African children. Our patient population was relatively homogenous, all having clinically defined CM. All children were treated on the same research unit for the duration of patient enrollment, decreasing the likelihood of any bias of results from differential sample collection or treatments received other than the anti-malarial administered. Complete data were available for $82 \%$ of patients who survived to hospital discharge. We found no demographic or clinical characteristics that were different between those who did and did not follow up at 1 month posthospitalization.

Like its predecessors, our study has several limitations. Our primary end point was measured by the collection of blood in

TABLE 5

Independent association of admission characteristics associated with post-admission hemolysis (multivariable logistic regression), adjusted for anti-malarial treatment

\begin{tabular}{lc}
\hline \multicolumn{1}{c}{ Admission characteristic $^{*}$} & Odds ratio $(95 \% \mathrm{Cl} \dagger)$ \\
\hline Hematocrit at admission & $10.11(3.21,35.41)$ \\
Ln(qHRP2) & $0.13(0.03,0.47)$ \\
Anti-malarial used (artesunate vs. quinine) & $3.23(0.88,18.38)$ \\
Interaction of Ln(qHRP2) with anti- & $6.02(1.13,39.04)$
\end{tabular}

Interaction of Ln(qHRP2) with antimalarial used

*Quantitative histidine-rich protein 2 (qHRP2) was fit in the natural logarithm scale. Ln(qHRP2) and hematocrit at admission were each rescaled (subtract overall mean and divide by $2 \mathrm{SDs}$ ) to be in the same scale to each other and to dichotomous covariates. †Ninety-five percent $\mathrm{Cl}$ with bootstrap. 
hematocrit tubes, centrifugation, and reading by a laboratory technician on a Micro Haematocrit Reader. Although our research laboratory has strict quality control standards, it is possible that readings from different technicians produced non-differential misclassification, potentially biasing results towards the null. It should be noted, however, that this methodology was also used in other studies conducted in Africa. ${ }^{10,11}$ Confirmation of hemolysis (and bone marrow response to it) requires determination of blood lactate dehydrogenase and haptoglobin levels; these analyses were not possible in the context of our study. Although our follow-up rate for hospital survivors was high, it was incomplete. It is possible that some children who survived hospitalization may have died before their 1-month follow-up appointment, and these deaths could have been caused by post-malaria anemia. The Malawi Ministry of Health recommends switching to enteral artemisinin combination therapy after three doses of parenteral artesunate, at variance with the WHO recommendation to continue artesunate until the patient can take medications orally. Some of our patients, because of the nature of their post-illness neurological impairments, are not able to take oral medications for extended periods of time (weeks), making strict adherence to the WHO recommendations problematic. This variance may have decreased our study population's exposure to intravenous artesunate, compared with patients hospitalized at other sites. Last, although patients treated with quinine and artesunate differed on measured co-variables (Table 1) and we were able to control for these differences in multivariate models, it is possible that unmeasured differences between the treatment groups may have further modified the resulting strength of association between exposure and outcome found in our analyses.

In summary, post-malaria anemia is rare in African children with $\mathrm{CM}$. It is equally common when children are treated with quinine or artesunate. A higher admission hematocrit value, lower qHRP2 level, and the presence of splenomegaly are risk factors for the development of post-malaria anemia in children treated with quinine and artesunate for cerebral malaria.

Received December 31, 2020. Accepted for publication February 24, 2021.

Published online April 26, 2021.

Note: Supplemental table appears at www.ajtmh.org.

Acknowledgment: We thank the staff of the Blantyre Malaria Project, whose clinical care of children with cerebral malaria made this study possible.

Financial support: G. G.'s work was supported by the Robert H. Parrott Research, Education, Advocacy, and Child Health Care Program at Children's National as well as by the Children's National Global Health Initiative. R. I.'s work in the Division of Biostatistics and Study Methodology was supported by award number UL1TR001876 from the NIH National Center for Advancing Translational Sciences. Financial support for statistical analysis was provided by intramural funds from the Division of Neurology at Children's National Medical Center.

Authors' addresses: Geoffrey Guenther, Department of Pediatrics, Children's National Medical Center, Washington, DC, E-mail: gguenther2@childrensnational.org. Alexuse M. Saidi, Blantyre Malaria Project, University of Malawi College of Medicine, Blantyre, Malawi, E-mail: alexmsaidi@gmail.com. Rima Izem, Division of Biostatistics and Study Methodology, Children's National Research Institute, Washington, DC, and Division of Epidemiology, The George Washington University School of Public Health, Washington, DC, E-mail: izem.rima@gmail.com. Karl Seydel, Blantyre Malaria Project, University of Malawi College of Medicine, Blantyre, Malawi, and Department of Osteopathic Medical Specialties, College of Osteopathic Medicine, Michigan State University, East Lansing, MI, E-mail: seydel@msu.edu. Douglas G. Postels, Blantyre Malaria Project, University of Malawi College of Medicine, Blantyre, Malawi, and Division of Neurology, The George Washington University/Children's National Medical Center, Washington, DC, E-mail: dpostels@childrensnational.org.

\section{REFERENCES}

1. World Health Organization, 2019. World Malaria Report 2019. Geneva, Switzerland: WHO. Available at: https://www.who.int/ publications/i/item/world-malaria-report-2019. Accessed August 8, 2020.

2. Dondorp A, Nosten F, Stepniewska K, Day N, White N, South East Asian Quinine Artesunate Malaria Trial (SEAQUAMAT) Group, 2005. Artesunate versus quinine for treatment of severe falciparum malaria: a randomised trial. Lancet 366: 717-725.

3. Dondorp AM et al., 2010. Artesunate versus quinine in the treatment of severe falciparum malaria in African children (AQUAMAT): an open-label, randomised trial. Lancet 376: 1647-1657.

4. Rehman K, Lötsch F, Kremsner PG, Ramharter M, 2014. Haemolysis associated with the treatment of malaria with artemisinin derivatives: a systematic review of current evidence. Int $J$ Infect Dis 29: 268-273.

5. Roussel C, Caumes E, Thellier M, Ndour PA, Buffet PA, Jauréguiberry S, 2017. Artesunate to treat severe malaria in travellers: review of efficacy and safety and practical implications. J Travel Med 24: 1-9.

6. Jauréguiberry S et al., 2014. Postartesunate delayed hemolysis is a predictable event related to the lifesaving effect of artemisinins. Blood 124: 167-175.

7. Rolling $T$ et al., 2014. Delayed hemolysis after treatment with parenteral artesunate in African children with severe malaria: a double-center prospective study. J Infect Dis 209: 1921-1928.

8. Scheu K et al., 2019. Determinants of post-malarial anemia in African children treated with parenteral artesunate. Sci Rep 9: 18134.

9. Hawkes MT et al., 2020. Anemia and transfusion requirements among Ugandan children with severe malaria treated with intravenous artesunate. Pediatr Hematol Oncol 37: 140-152.

10. Varo R et al., 2020. Post-malarial anemia in Mozambican children treated with quinine or artesunate: a retrospective observational study. Int J Infect Dis 96: 655-662.

11. Fanello $C$ et al., 2017. Post-treatment haemolysis in African children with hyperparasitaemic falciparum malaria: a randomized comparison of artesunate and quinine. BMC Infect Dis 17: 575.

12. World Health Organization, Regional Office for the Western Pacific, 2016. Malaria Microscopy Standard Operating Procedures. Available at: https://apps.who.int/iris/handle/10665/ 274382. Accessed April 2, 2021.

13. Weimer A et al., 2019. Blood transfusion safety in sub-Saharan Africa: a literature review of changes and challenges in the $21 \mathrm{st}$ century. Transfusion 59: 412-427.

14. Kabaghe A et al., 2017. Short-term changes in anemia and malaria parasite prevalence in children under 5 years during one year of repeated cross-sectional surveys in rural Malawi. Am J Trop Med Hyg 97: 1568-1575. 